\title{
Longitudinal painting with large amplitude second harmonic rf voltages in the rapid cycling synchrotron of the Japan Proton Accelerator Research Complex
}

\author{
Fumihiko Tamura, ${ }^{*}$ Masanobu Yamamoto, Masahito Yoshii, Chihiro Ohmori, Masahiro Nomura, Alexander Schnase, \\ Makoto Toda, Hiromitsu Suzuki, Taihei Shimada, Keigo Hara, and Katsushi Hasegawa \\ J-PARC Center, KEK \& JAEA, 2-4 Shirakata-Shirane, Tokai, Naka, Ibaraki, Japan 319-1195
}

(Received 20 November 2008; published 14 April 2009)

\begin{abstract}
In the rapid cycling synchrotron (RCS) of the Japan Proton Accelerator Research Complex (J-PARC), the longitudinal painting is important to alleviate the space-charge effects. It is known that the momentum offset injection and applying the second harmonic rf voltage improves the bunching factor, which is defined as the ratio of average and peak current. Our simulation studies show that the large-amplitude second harmonic, $80 \%$ to the fundamental, is optimum, and the second harmonic phase sweep improves the bunching factor at the beginning of the injection period. We performed the beam tests of longitudinal painting in the J-PARC RCS. We proved that the longitudinal painting with the $80 \%$ second harmonic, the momentum offset of $-0.2 \%$, and the second harmonic phase sweep improved bunching factors significantly.
\end{abstract}

DOI: 10.1103/PhysRevSTAB.12.041001

PACS numbers: 29.20. $-\mathrm{c}, 29.27 .-\mathrm{a}$

\section{INTRODUCTION}

The beam power of the $3 \mathrm{GeV}$ rapid cycling synchrotron (RCS) of the Japan Proton Accelerator Research Complex (J-PARC) [1,2] is $1 \mathrm{MW}$ by design. For acceleration of the high beam current with minimum beam losses, it is essential to suppress the space-charge effects as much as possible. The transverse painting scheme is employed in the RCS during the charge-exchange multiturn injection. By the painting scheme, the beam distribution in the phase space is widened to alleviate the space-charge tune shift. The transverse painting is realized by programing the bump magnet system. In addition, longitudinal painting [3] is employed during injection by adjusting the rf system parameters and programs.

The bunching factor $\left(B_{f}\right)$, which is defined as the ratio,

$$
B_{f}=\frac{\text { (average current) }}{\text { (peak current) }}
$$

is the key parameter of the longitudinal painting. By increasing the bunching factor we can reduce the spacecharge tune shift.

The following painting methods are widely known: (i) the momentum offset injection, and (ii) applying the second harmonic rf voltage to generate "flat" rf buckets. At the CERN Proton Synchrotron Booster, the second harmonic rf system is used in normal operation to generate flattopped bunches [4]. In Brookhaven the AGS booster employs the second harmonic of for the bunch shape control [5]. Also it is reported [6] that the stability threshold is increased nearly $10 \%$ with the second harmonic rf voltages in the RCS of the intense pulsed neutron source at Argonne National Laboratory. In the ISIS synchrotron, the second

\footnotetext{
*fumihiko.tamura@j-parc.jp
}

harmonic rf cavities have been installed and operated [7]. Extensive simulation studies have been performed for the injection to the Fermilab main injector [8,9].

We combine the momentum offset method and the second harmonic rf for the longitudinal painting in JPARC RCS. We also employ the chopped beam injection to reduce the beam loss during the injection period. By exploring the parameters with the longitudinal particle tracking simulations, the large amplitude of the second harmonic rf, $80 \%$ to the fundamental rf, has been chosen, while flat $\mathrm{rf}$ bucket shapes are obtained by the $50 \%$ ratio. Also, to modify the bucket shape during the injection period, the "second harmonic phase sweep" method, which sweeps the relative phase of the second harmonic to the fundamental $\mathrm{rf}$, has been proposed. The details of the simulation studies are described in [10] and [2], pp. 128139. The longitudinal simulation code is written by one of the authors, Yamamoto. The code includes the space charge and the multiharmonic beam loading. The simulation studies show that the improved bunching factor can approach 0.4 after the injection by combination of the above methods. In Fig. 1, a typical bunch shape simulated using these schemes is illustrated. In the simulation, the second harmonic voltage ratio to the fundamental is $80 \%$ at maximum.

The parameters of the rf system of the RCS are listed in Table I [11]. The proton beam in the RCS is accelerated from $181 \mathrm{MeV}$ to $3 \mathrm{GeV}$ in $20 \mathrm{~ms}$ with a repetition rate of $25 \mathrm{~Hz}$. This rapid acceleration needs strong acceleration voltages, the maximum rf voltage is $450 \mathrm{kV}$ per turn. The harmonic number is chosen as 2 and the accelerating frequency sweeps from 0.938 to $1.671 \mathrm{MHz}$.

We employ magnetic-alloy (MA) loaded cavities to generate the high field-gradient accelerating voltage in the limited space of the RCS ring. The $\mathrm{Q}$ value of the $\mathrm{rf}$ 

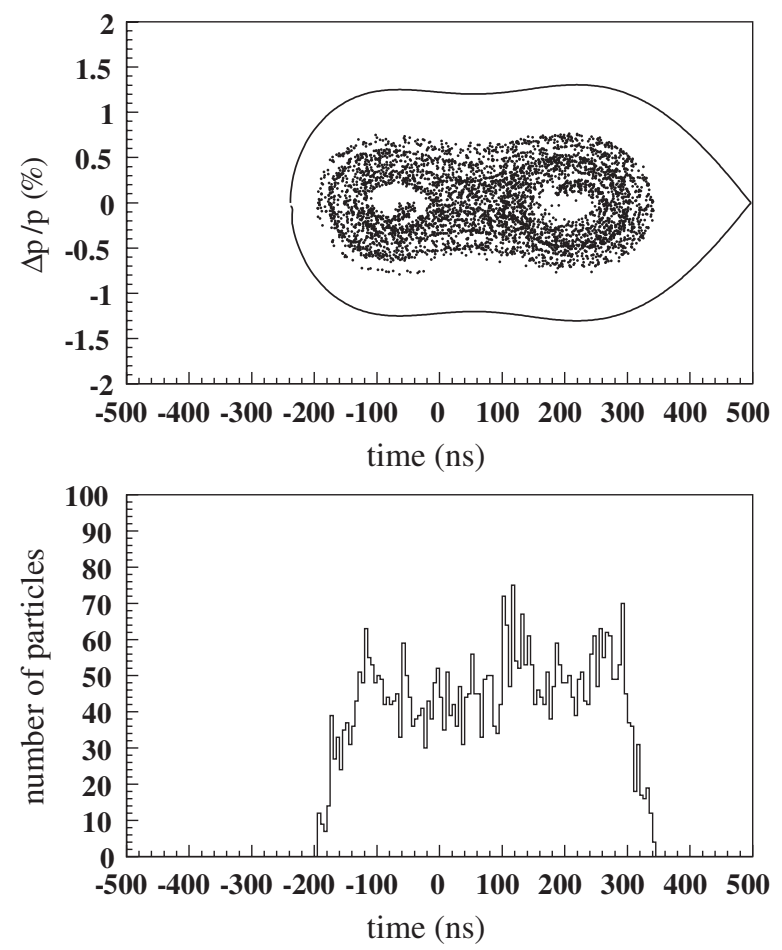

FIG. 1. A typical bunch shape after injection applying the second harmonic rf at the 1000th turn (beginning of the acceleration). The ratio of the second harmonic to the fundamental is $80 \%$.

cavity is adjusted to be 2 . The bandwidth is wide enough to cover the accelerating frequency sweeps without tuning loops. We have chosen the bandwidth so that the dualharmonic operation is fully supported. In the dualharmonic operation, each single cavity is driven by a superposition of the rf signals, the fundamental accelerating $\mathrm{rf}(h=2)$ and the second harmonic $\mathrm{rf}(h=4)$. The second harmonic rf modifies the rf bucket shape, and is used for the longitudinal bunch shape control and longitudinal painting. By the dual-harmonic operation, it is possible to generate large-amplitude second harmonic rf voltages without extra cavities for the second harmonic. To realize the dual-harmonic operation a dual-harmonic

TABLE I. Parameters of the J-PARC RCS and its rf system.

\begin{tabular}{lc}
\hline \hline Circumference & $348.333 \mathrm{~m}$ \\
\hline Energy & $0.181-3 \mathrm{GeV}$ \\
Accelerating frequency & $0.938-1.671 \mathrm{MHz}$ \\
Harmonic number & 2 \\
Maximum rf voltage & $450 \mathrm{kV}$ \\
Repetition & $25 \mathrm{~Hz}$ \\
Duty (power) & $30 \%$ \\
Number of cavities & $11^{\mathrm{a}}$ \\
Q value & 2 \\
\hline \hline
\end{tabular}

${ }^{\text {a }}$ This is the design number. The day-1 operation started with ten cavities. auto voltage control (AVC), which controls the fundamental and the second harmonic voltages independently, is necessary. We have designed and built the dual-harmonic $\mathrm{AVC}$ as a part of the digital low level rf (LLRF) control system of the J-PARC RCS [12]. The dual-harmonic AVC is working properly.

Thus, thanks to the dual-harmonic operation with the wideband MA loaded cavity and the precise dual-harmonic $\mathrm{AVC}$, it is possible to generate large amplitude ( $80 \%$ to the fundamental rf) second harmonic rf voltages.

We performed the beam tests of the longitudinal painting in the J-PARC RCS. The test results are described in Sec. II. The summary and the discussions are in Sec. III.

\section{BEAM TEST RESULTS}

The J-PARC RCS has two modes of beam operation for circulating beam tuning, (i) beam storage mode and (ii) acceleration mode. In the beam storage mode, the RCS is operated like a storage ring at the injection energy. In the acceleration mode, the RCS accelerates the injected beam at the energy of $181 \mathrm{MeV}$ up to $3 \mathrm{GeV}$ in $20 \mathrm{~ms}$. We have performed the beam tests of the longitudinal painting injection in both modes.

The momentum offset injection is realized by applying a frequency offset corresponding to the momentum offset. The frequency offset $d f / f$ is given as

$$
\frac{d f}{f}=\eta \times \frac{d p}{p}
$$

where $\eta$ is the slippage, and $d p / p$ the momentum offset. In case of the RCS injection, $\eta=-0.69$.

Since the direct digital synthesis (DDS) is employed [12], the rf frequency is accurate and stable, in the order of $0.1 \mathrm{ppm}$ or $0.1 \mathrm{~Hz}$. Thus, the momentum offset error is very small. At the injection, $0.1 \mathrm{~Hz}$ corresponds to the momentum offset of $1.5 \times 10^{-7}$. The error of the voltage

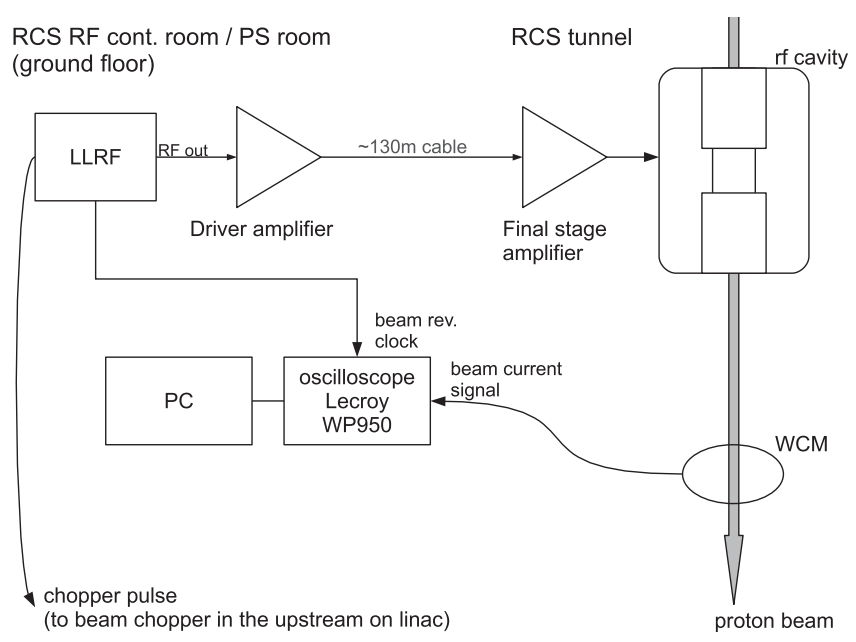

FIG. 2. Beam test setup. For simplicity, only one of the ten cavities is shown. 


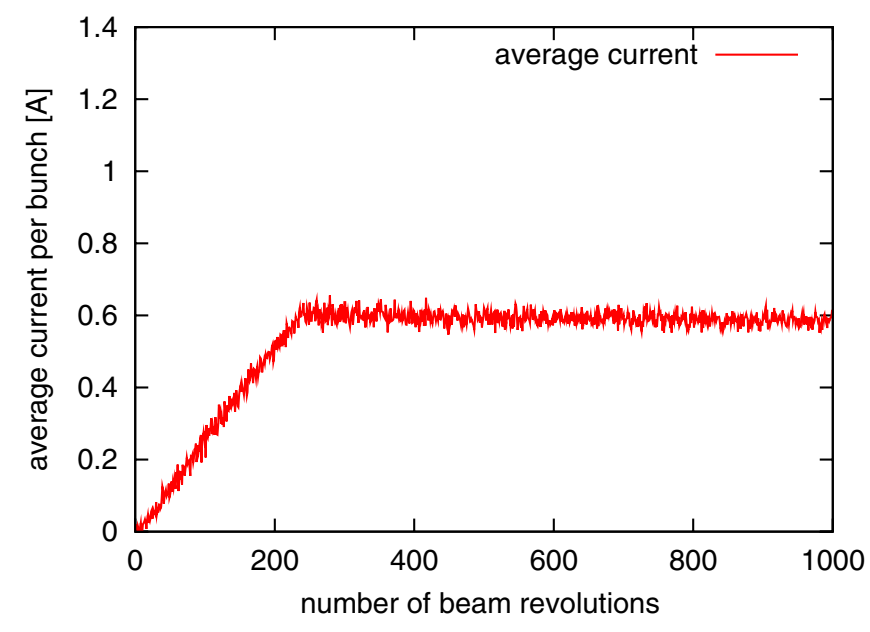

FIG. 3. (Color) Typical average current per bunch up to 1000th turn. The beam from the linac is injected for 234 turns. This is equivalent to $500 \mu \mathrm{s}$ at the revolution frequency of $469 \mathrm{kHz}$ during the injection period.

ratio between the fundamental and the second harmonic rf is about $5 \%$.

The beam test setup is illustrated in Fig. 2. The beam current signal is picked up by a wall current monitor (WCM) and recorded by an oscilloscope (Lecroy WP950) at the sample rate of 200 Msamples/s. The recorded signals are analyzed with the beam revolution clock generated by the LLRF control system.

The RCS employs the chopped beam injection. The timing of the beam chopper at the upstream of the linac is controlled by the chopper pulse, which is generated by the LLRF control system of the RCS. The chopping width is one of the key parameters of the longitudinal painting.

The typical average current per bunch near the injection period is plotted in Fig. 3. The macropulse width of the linac beam is $500 \mu \mathrm{s}$, which corresponds to 234 turns at the injection frequency.

\section{A. Beam storage mode}

The beam test in the beam storage mode was performed in June 2008. In the beam storage mode, the measurement and analysis are more straightforward than in the acceleration mode; for example, the rf frequency is fixed and the $\mathrm{rf}$ bucket is stationary. We performed the beam test in the beam storage to explore the painting parameters.

The beam test conditions are listed in Table II. We performed the test in the one-bunch operation, in which one of two rf buckets is filled.

The mountain plots and the bunching factor up to 1000th turn with the following parameters are shown in Figs. 4 and 5 , respectively: (i) fundamental rf only; (ii) with the second harmonic at the amplitude of $50 \%$ of the fundamental; (iii) with the second harmonic at the amplitude of 50\% and the momentum offset of $-0.2 \%$; (iv) with the second harmonic at the amplitude of $80 \%$ of the fundamental; (v) with the second harmonic at the amplitude of $80 \%$ and the momentum offset of $-0.2 \%$. The momentum offset of $-0.2 \%$ corresponds to the frequency offset of $+2512 \mathrm{~Hz}$.

In the mountain plots, the vertical axis is the number of the turns after injection and the horizontal axis is the time in each slice in nanosecond. The color code corresponds to the WCM signal height, i.e., beam intensity. The WCM signal has a negative offset because of the AC-coupling circuit.

The charge density at the center of the rf bucket was high in the case without the second harmonic [see Figs. 4 (1) and 5 (1)]. The bunching factor after injection was about 0.2 . Applying the second harmonic rf voltages of 50\% to the fundamental, one can see that the bunch was flattened. The bunching factor was improved to about 0.25 [Figs. 4 (2)

TABLE II. Beam test parameters.

\begin{tabular}{|c|c|c|}
\hline & Storage mode & Acceleration mode \\
\hline Macropulse width & $500 \mu \mathrm{s}^{\mathrm{a}}$ & $500 \mu \mathrm{s}$ \\
\hline Chopping width & $560 \mathrm{~ns}$ & $560 \mathrm{~ns}$ \\
\hline Linac peak current & $5 \mathrm{~mA}$ & $10 \mathrm{~mA}$ \\
\hline Number of filled buckets & 1 & 2 \\
\hline Number of accelerated protons & $4 \times 10^{12}$ (one bunch) & $1.4 \times 10^{13}$ (two bunches) \\
\hline Momentum spread of linac beam & $\pm 0.05 \%$ & $\pm 0.05 \%$ \\
\hline Fundamental $\mathrm{rf}$ frequency during injection ${ }^{\mathrm{b}}$ & $0.938 \mathrm{MHz}$ & $0.938-0.939 \mathrm{MHz}$ (programed) \\
\hline Fundamental rf voltage during injection & $68 \mathrm{kV}$ (fixed) & 78-111 kV (programed) \\
\hline Relative bucket height during injection ${ }^{\mathrm{c}}$ & $0.97 \%$ & $1.03 \%-1.24 \%$ \\
\hline Synchrotron frequency during injection ${ }^{c}$ & $3146 \mathrm{~Hz}$ & $3370-4009 \mathrm{~Hz}$ \\
\hline Phase feedback & Off & On \\
\hline
\end{tabular}

${ }^{\mathrm{a}}$ It corresponds to 234 turns (see Fig. 3).

${ }^{\mathrm{b}}$ Without the momentum offset.

${ }^{\mathrm{c}}$ Without the second harmonic rf. 

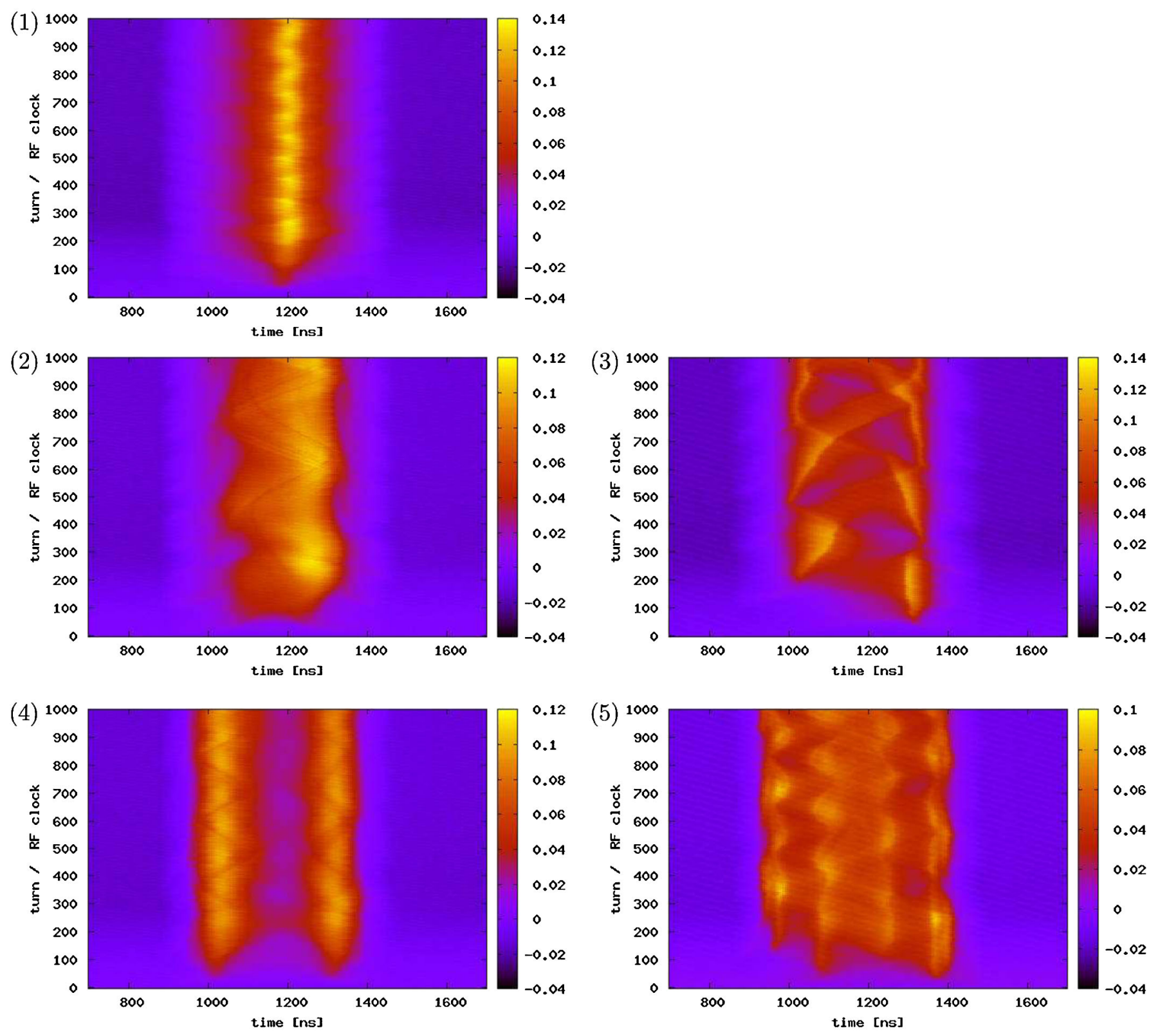

FIG. 4. (Color) Mountain plots of the longitudinal beam signal. (upper left) (1) Fundamental rf only; (middle left) (2) with 50\% second harmonic to the fundamental; (middle right) (3) with $50 \%$ second harmonic and momentum offset of $-0.2 \%$; (lower left) (4) with $80 \%$ second harmonic; (lower right) (5) with $80 \%$ second harmonic and momentum offset of $-0.2 \%$.

and 5 (2)]. Comparing (2) and (3) in Fig. 5, adding the momentum offset had little effect for further improving the bunching factor in the case of the 50\% second harmonic.

In the case of applying the second harmonic of $80 \%$ without momentum offset [Figs. 4 (4) and 5 (4)], the bunch shape had two peaks. The reason for this is that the rf bucket is no longer flat but shows two peaks with the $80 \%$ second harmonic. The bunching factor was improved, about 0.27 .

Applying the momentum offset of $-0.2 \%$ in addition to the second harmonic, it is demonstrated that the bunch shape became flat and the bunching factor became about
0.33, it was further improved [Figs. 4 (5) and 5 (5)]. We tried the momentum offset from $-0.1 \%$ down to $-0.7 \%$, and $-0.2 \%$ was the best in terms of both the bunching factor and the bunch shape. However, one can notice that the bunching factor decreases near the 50th turn down to 0.15 and the recovery to the higher level is slow. This reduction corresponds to a $1 / 4$ turn of the synchrotron motion, since the rf bucket is not matched to the injected beam from the linac with a narrow momentum spread. By applying the second harmonic phase sweep during the injection period, we could suppress the reduction and improve the recovery time of the bunching factor. In 

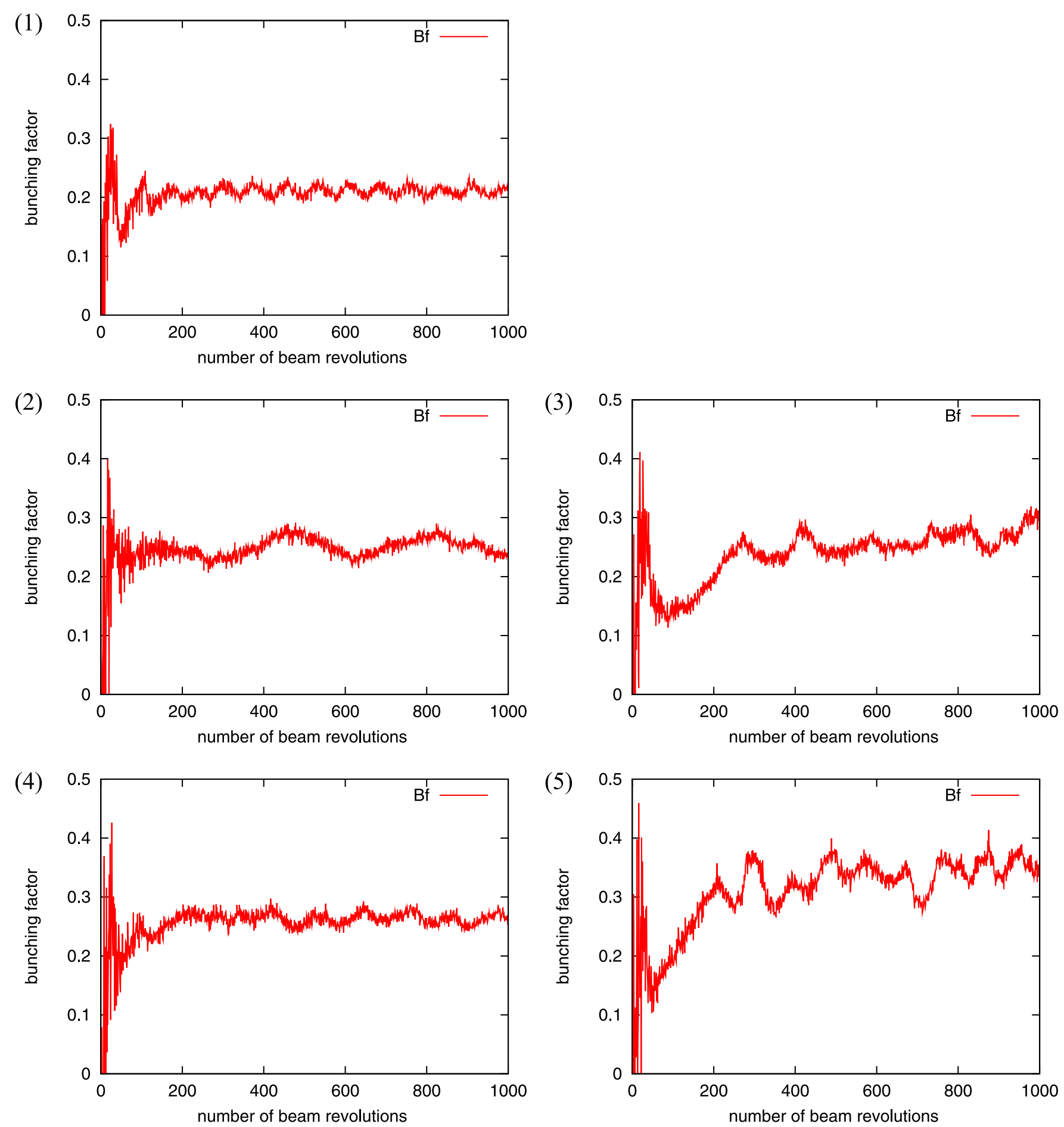

FIG. 5. (Color) Bunching factor plots corresponding to Fig. 4. (upper left) (1) Fundamental rf only; (middle left) (2) with 50\% second harmonic to the fundamental; (middle right) (3) with 50\% second harmonic and momentum offset of $-0.2 \%$; (lower left) (4) with $80 \%$ second harmonic; (lower right) (5) with $80 \%$ second harmonic and momentum offset of $-0.2 \%$.

Fig. 6 the mountain plot and the bunching factor plot with the second harmonic phase sweep of 60 degrees in addition to the $80 \%$ second harmonic and the momentum offset of $-0.2 \%$ are shown. The second harmonic phase relative to the fundamental was swept as

$$
\phi_{(h=4)}=\frac{\phi_{\text {sweep }}}{T_{\text {inj }}}\left(t-\frac{T_{\text {inj }}}{2}\right)-2 \phi_{s}[\mathrm{deg}]
$$

where $\phi_{(h=4)}$ is the second harmonic phase, $\phi_{\text {sweep }}$ the sweep range that was set to 60 degrees, $T_{\mathrm{inj}}$ the duration 

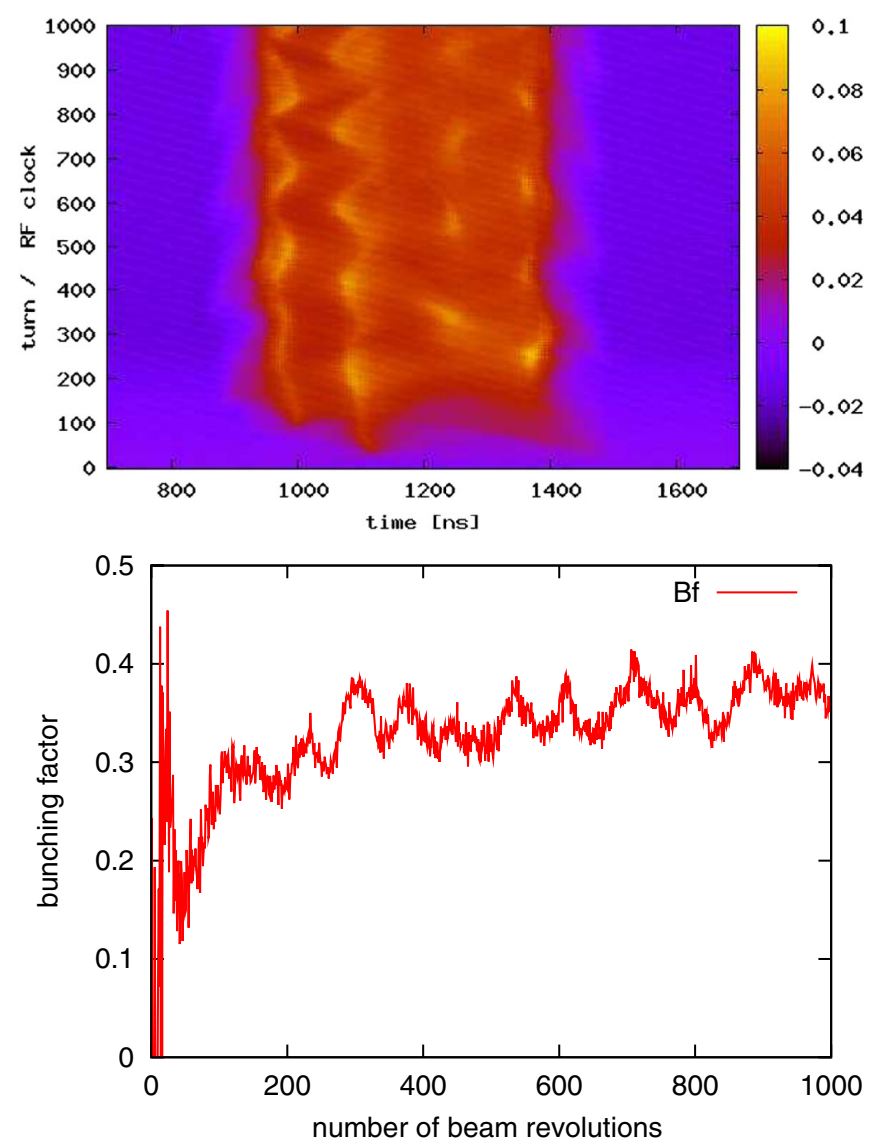

FIG. 6. (Color) (Upper) Mountain plot and (lower) bunching factor plot, with $80 \%$ second harmonic, momentum offset of $-0.2 \%$, and phase sweep of 60 degrees.

of the injection, and $\phi_{s}$ the synchronous phase of the beam. In the beam storage mode $\phi_{s}=0$. The bunching factor reduction became only a bit smaller, 0.17 at the minimum; however, the recovery became much faster than the case without the second harmonic phase sweep. Thus, the second harmonic phase sweep improves the transient behavior

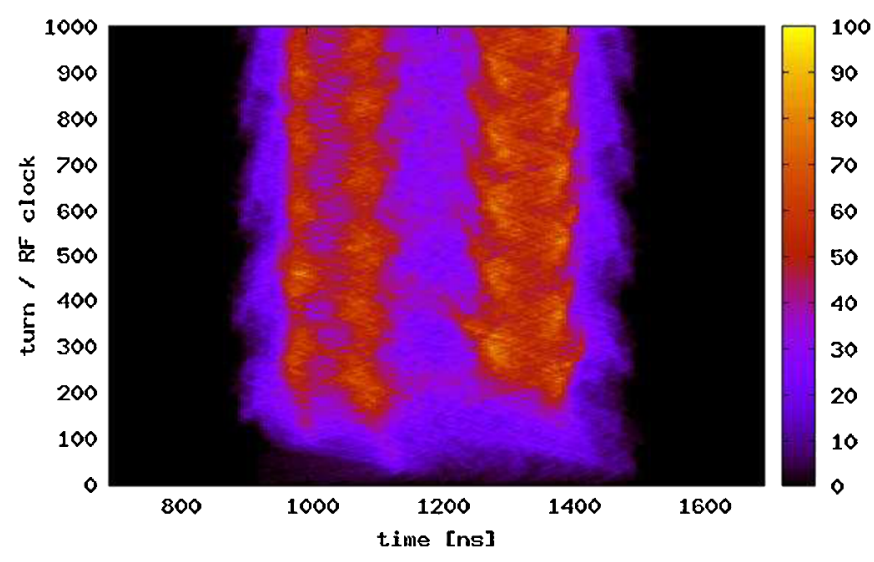

FIG. 7. (Color) Mountain plots of the simulated beam, second harmonic $80 \%$, momentum offset of $-0.2 \%$, and phase sweep of 60 degrees.

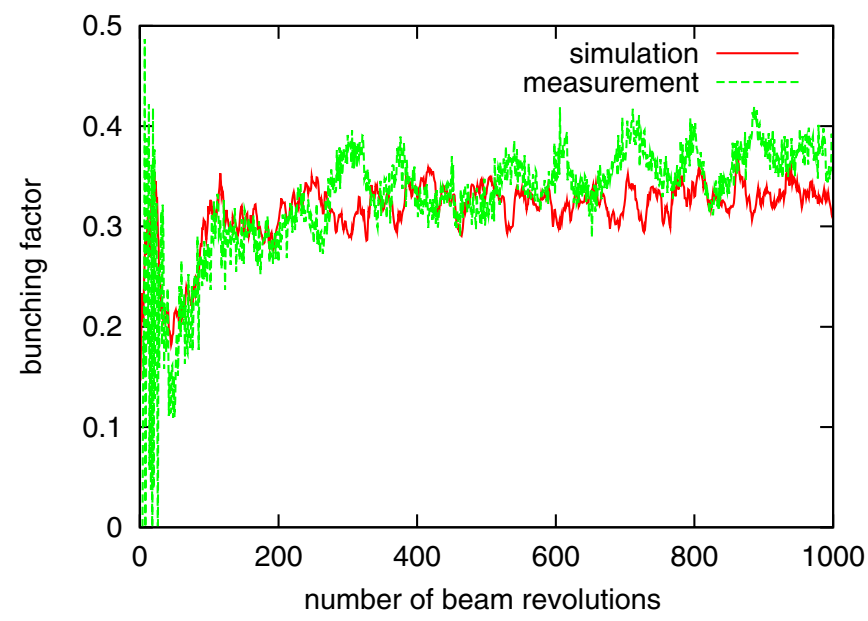

FIG. 8. (Color) Comparison between the simulation and measurement, with $80 \%$ second harmonic, momentum offset of $-0.2 \%$, and phase sweep of 60 degrees.
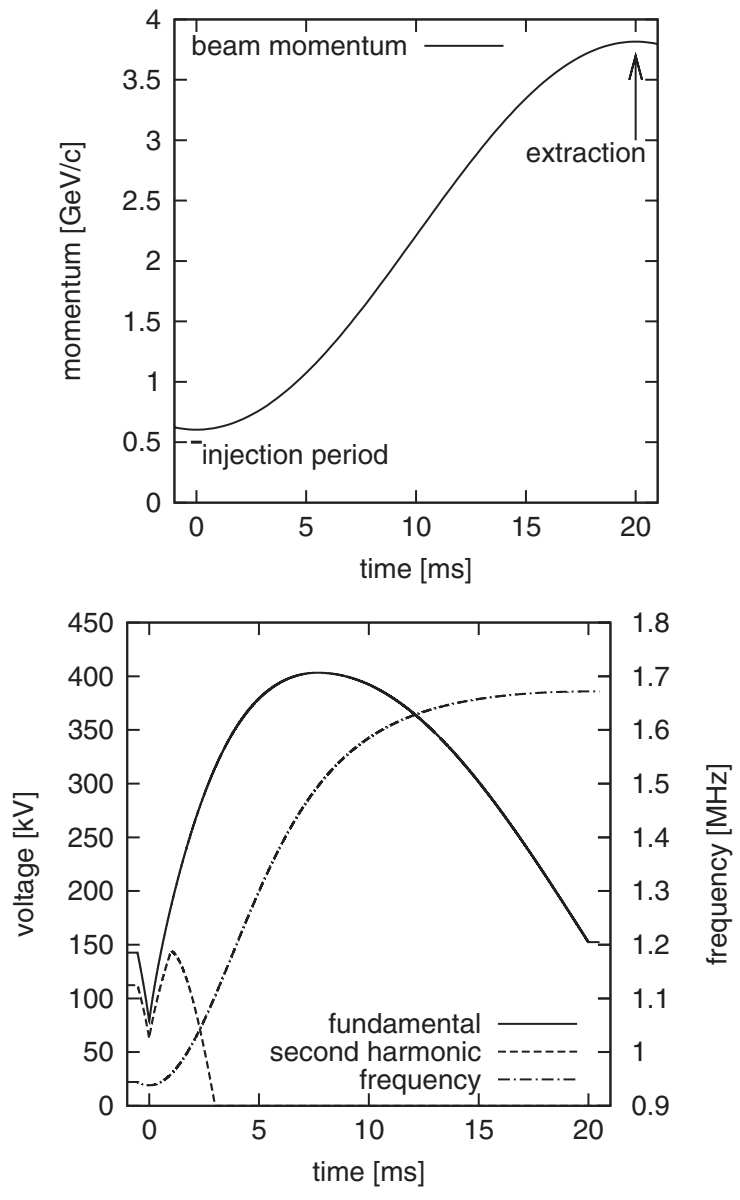

FIG. 9. (upper) Beam momentum in the accelerating cycle. (lower) Accelerating voltage program with $80 \%$ second harmonic and frequency program. $t=0$ corresponds to $B_{\min }$. The beam is injected from 117 turns before $B_{\min }$ to 117 turns after $B_{\min }$. 
of the bunching factor. The mountain plot became asymmetric because the rf bucket becomes asymmetric with the second harmonic phase sweep. The bunching factor after the 250th turn did not change.

The mountain plot of the simulated beam with the same conditions is shown in Fig. 7. The bunching factor comparison is shown in Fig. 8. This simulation is carried out with space-charge effects. The mountain plots of the simulation and the measurement are similar. Also, the simulation and the measurement agree in terms of the bunching factor.

\section{B. Acceleration mode}

The beam test in the acceleration mode was performed in September 2008. The beam conditions are listed in Table II. The accelerating voltage program is shown in Fig. 9. In the acceleration mode, the second harmonic rf is superposed at the maximum ratio from the beginning of the injection to $1 \mathrm{~ms}$ after the minimum value of the bending magnet field $\left(B_{\min }\right)$, and the ratio is linearly reduced. At $3 \mathrm{~ms}$ after $B_{\min }$, the amplitude of the second harmonic voltage goes to zero.

The beam is injected from 117 turns before $B_{\min }$ to 117 turns after $B_{\min }$. This is equivalent to $500 \mu \mathrm{s}$ at the revolution frequency of $469 \mathrm{kHz}$ during the injection period.
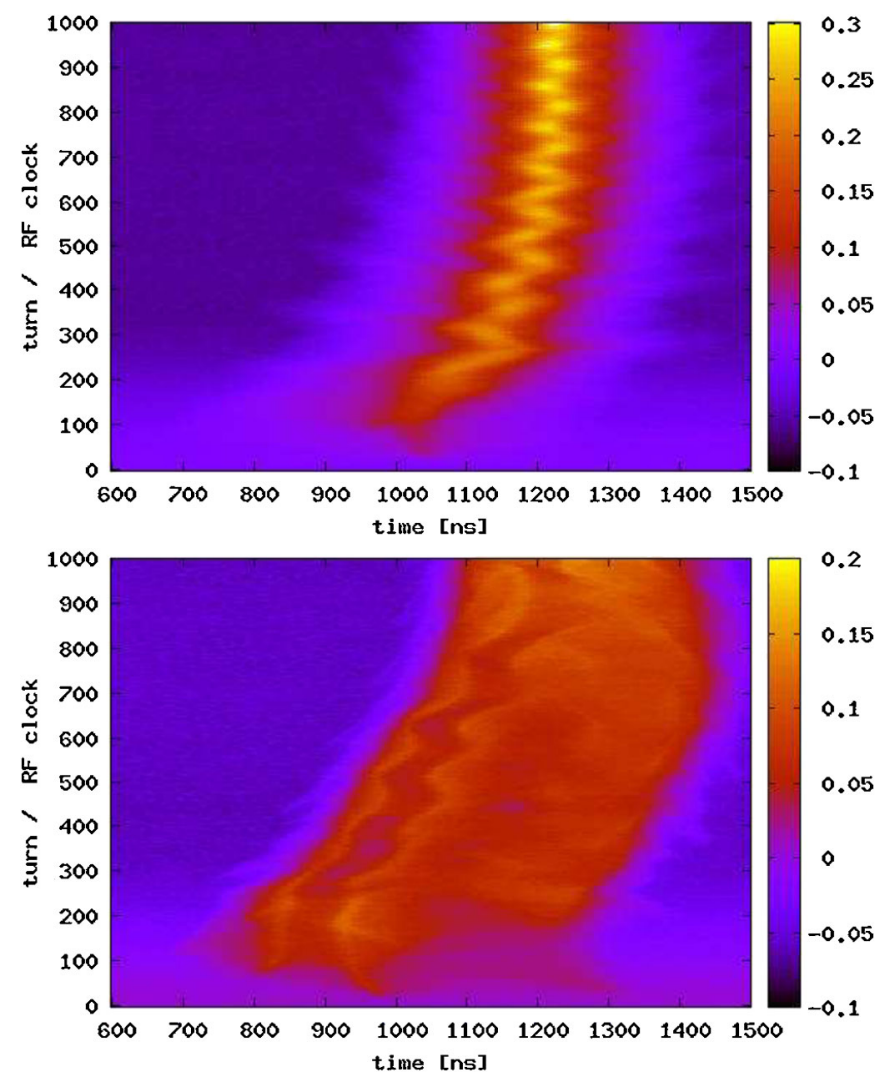

FIG. 10. (Color) Mountain plots: (upper) fundamental rf only; (lower) with $80 \%$ second harmonic, momentum offset of $-0.2 \%$, and phase sweep of 80 degrees.
The mountain plots of the case with only fundamental rf and no momentum offset and the case with the $80 \%$ second harmonic, the momentum offset of $-0.2 \%$, and the second harmonic phase sweep of 80 degrees are shown in Fig. 10. The bunching factor plots and the bunch shapes are in Figs. 11 and 12, respectively. In the acceleration mode, the second harmonic phase sweep of 80 degrees showed a better bunching factor compared to 60 degrees, which was used for the study in the beam storage mode.

We demonstrate that the bunch was widened and the bunching factor was improved with the longitudinal painting methods above. The bunching factor of about 0.37 was achieved just after injection (at 250th turn), while it was 0.21 in the case without longitudinal painting.

The mountain plot of the simulated beam with the same conditions, except the phase feedback loop, is shown in Fig. 13. The simulation is carried out with space-charge effect. The bunching factor comparison is shown in Fig. 14. In Fig. 14 the bunching factor without space-charge effects is also plotted. The two traces with and without space charge are quite similar. This means that the space-charge
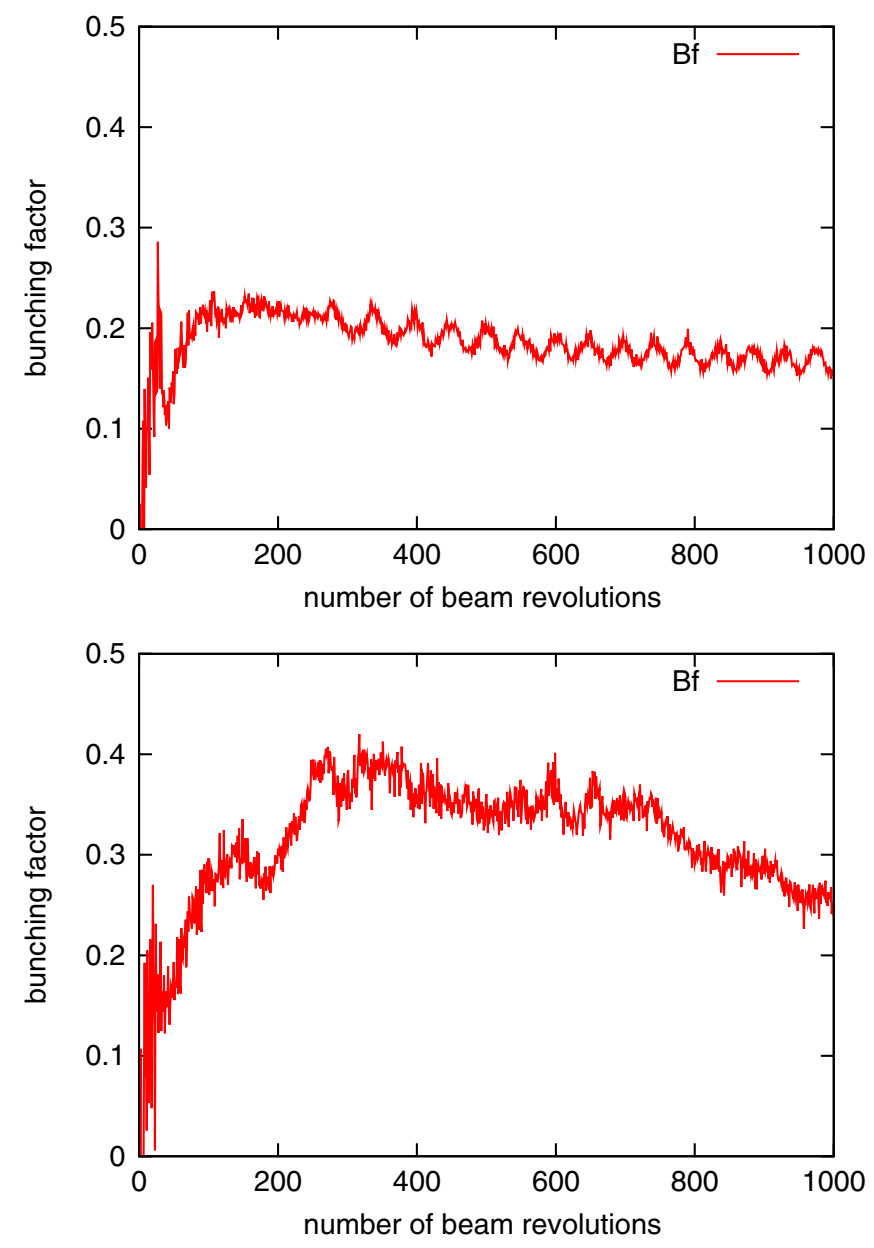

FIG. 11. (Color) Bunching factor plots: (upper) fundamental rf only; (lower) with $80 \%$ second harmonic, momentum offset of $-0.2 \%$, and phase sweep of 80 degrees. 

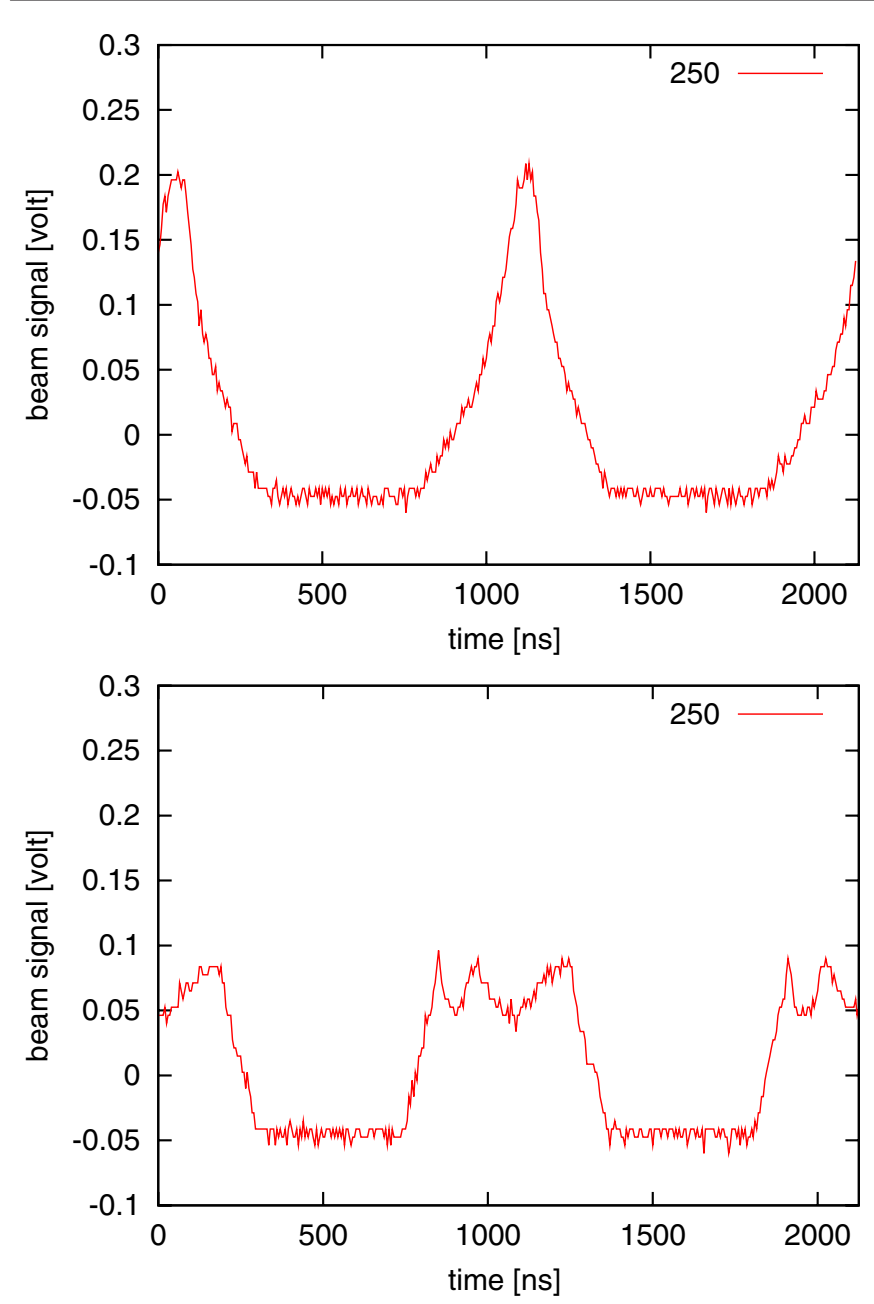

FIG. 12. (Color) Bunch shapes at 250th turn: (upper) fundamental rf only; (lower) with $80 \%$ second harmonic, momentum offset of $-0.2 \%$, and phase sweep of 80 degrees. The negative offset of the signal is due to the AC-coupling circuit.

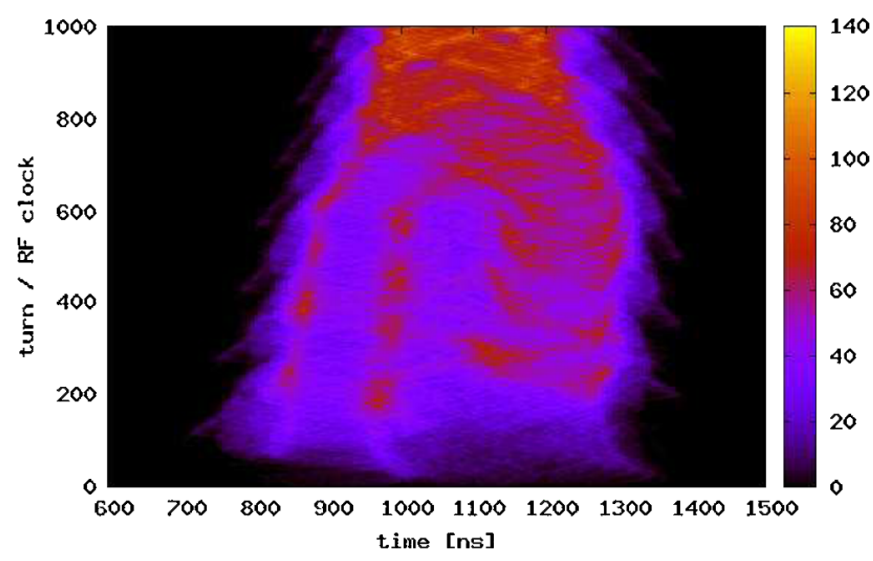

FIG. 13. (Color) Mountain plots of the simulated beam, with $80 \%$ second harmonic, momentum offset of $-0.2 \%$, and phase sweep of 80 degrees.

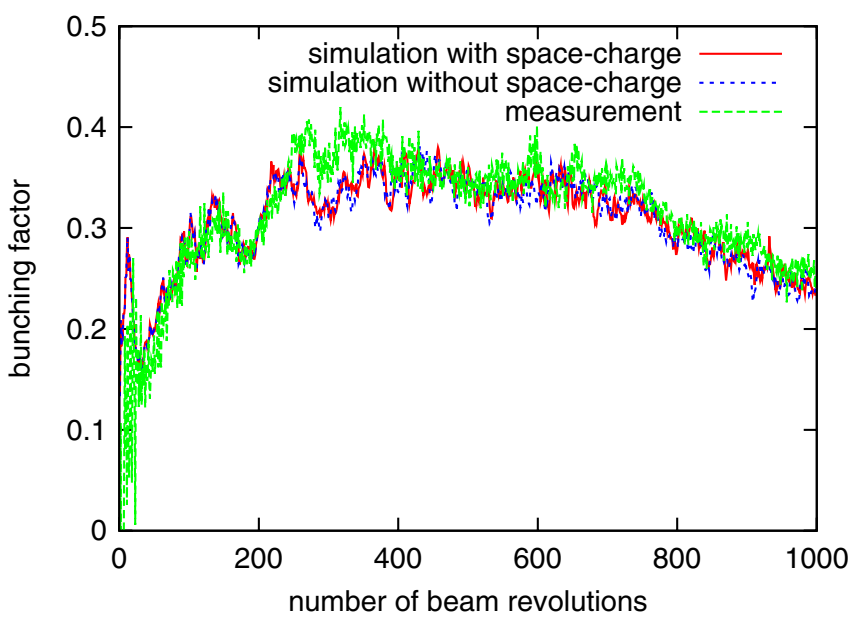

FIG. 14. (Color) Comparison between the simulation with/without space-charge effects and measurement, with $80 \%$ second harmonic, momentum offset of $-0.2 \%$, and phase sweep of 80 degrees.

effects on the longitudinal distribution are small at this beam intensity. The simulation and the measurement agree very well in terms of the bunching factor. The mountain plot of the measured beam signal shows an angle, which does not appear in the simulation. The angle is caused by the response of the closed phase loop. Except for the angle, the mountain plots of the measurement and the simulation look comparable. The simulation code works well.

In the beam tests we have proven that the longitudinal painting with large-amplitude second harmonic rf voltages improves the bunching factor significantly.

\section{SUMMARY AND DISCUSSIONS}

We summarize the article as follows: (i) In the J-PARC RCS, the longitudinal painting is essential as well as transverse painting to alleviate the space-charge effects. (ii) The bunching factor, which is defined by the ratio of average and peak current, is the key parameter. The momentum offset injection and applying the second harmonic rf voltage improves the bunching factor. The simulation studies show that the large-amplitude second harmonic, $80 \%$ to the fundamental, is optimum and the bunching factor near 0.4 is possible. Additionally, the second harmonic phase sweep improves the transient behavior of the bunching factor. (iii) The dual-harmonic operation, in which each single cavity is driven by a superposition of the fundamental and the second harmonic rf signals, is useful to generate a large-amplitude second harmonic rf voltage in the limited space in the RCS ring. The dualharmonic operation in the J-PARC RCS is possible thanks to the wideband MA loaded cavity and the digital LLRF including the dual-harmonic AVC. (iv) We performed the beam tests of longitudinal painting in the J-PARC RCS in both the beam storage mode and the acceleration mode. In both modes, the longitudinal painting with the $80 \%$ second 
harmonic, the momentum offset of $-0.2 \%$, and the second harmonic phase sweep showed significantly improved bunching factors. (v) The simulation and the measurement agree. The simulation code works well.

Applying the longitudinal painting at the beam intensity of $1.4 \times 10^{13}$ protons per pulse, we did not observe a significant reduction of the beam losses due to the space charge. In both cases with and without longitudinal painting, the beam transmission ratios from the injection to the extraction were more than 0.99 . The reason for the $1 \%$ loss is considered as resulting from charge-exchange foil hitting. We need more beam intensity to observe the effectiveness and usefulness of the longitudinal painting. We are going to perform the next beam tests with higher intensities.

Although the tests were performed with still lower intensities than the design value, the longitudinal painting parameters that will improve the bunching factors of the full intensity beams have been confirmed.

\section{ACKNOWLEDGMENTS}

We would like to thank Professor E. Ezura, Professor K. Takata, and Professor A. Ando for fruitful discussions and comments. The linac and the RCS provided the stable beam condition during the beam test of the longitudinal painting; we are grateful to all members of the J-PARC linac and the RCS.
[1] KEK-Report 97-16, 1997.

[2] JAERI-TECH 2003-044, 2003.

[3] Y. Kamiya, in Proceedings of 1989 Particle Accelerator Conference (PAC 89), 1989, pp. 660-662.

[4] J. M. Baillod et al., IEEE Trans. Nucl. Sci. 30, 3499 (1983).

[5] J. M. Brennan et al., in Proceedings of the 18th Particle Accelerator Conference, New York, 1999 (IEEE, New York, 1999), pp. 614-616.

[6] M.E. Middendorf et al., in Proceedings of the 2007 Particle Accelerator Conference, Albuquerque, New Mexico, 2007 (IEEE, Albuquerque, New Mexico, 2007), pp. 2233-2235.

[7] A. Seville et al., in Proceedings of the 11th European Particle Accelerator Conference, Genoa, 2008 (EPS-AG, Genoa, Italy, 2008), pp. 349-351.

[8] D. E. Johnson et al., in Proceedings of the 2007 Particle Accelerator Conference, Albuquerque, New Mexico, 2007 (Ref. [6]), pp. 1700-1702.

[9] P. S. Yoon et al., FERMILAB-TM-2398-AD-APC, 2008.

[10] M. Yamamoto et al., in Proceedings of the 8th European Particle Accelerator Conference, Paris, 2002 (EPS-IGA and CERN, Geneva, 2002), pp. 1073-1075.

[11] M. Yoshii et al., in Proceedings of the 11th European Particle Accelerator Conference, Genoa, 2008 (Ref. [7]), pp. 385-387.

[12] F. Tamura, A. Schnase, and M. Yoshii, Phys. Rev. ST Accel. Beams 11, 072001 (2008). 